\title{
EL TIEMPO DE MADARIAGA Y LA IDEA DE EUROPA: SALVADOR DE MADARIAGA EN EL CONGRESO DE EUROPA EN LA HAYA (1948)
}

\author{
THE TIME OF MADARIAGA AND THE IDEA OF EUROPE: \\ SALVADOR DE MADARIAGA \\ IN THE CONGRESS OF EUROPE IN THE HAGUE (1948) \\ Víctor Gavin Munté* \\ Universidad de Barcelona, España
}

\begin{abstract}
RESUMEN: La idea de Europa en Salvador de Madariaga se fundamentaba en el convencimiento que, sin la creación previa de una conciencia europea, entendida como la capacidad de pensar los problemas de Europa sin tomar en cuenta las fronteras que dividen a los diferentes estados que la conforman, cualquier proyecto de integración política o económica estaría incompleto. La solidaridad de hecho, es decir, aquella derivada de las inevitables interrelaciones político-económicas entre los estados, debería completarse con la solidaridad subjetiva que une a las personas más allá de los intereses materiales, convirtiendo así sus problemas en comunes sólo solucionables con planes también comunes. Ello sería posible con la existencia de una conciencia europea que debería ser asumida por una élite encargada de dotar de «alma europea» a las instituciones que se crearan. Esta idea la defenderá Salvador de Madariaga en el Congreso de Europa en La Haya (1948) y tendrá su plasmación práctica en el Colegio de Europa en Brujas (1949) fundado a iniciativa suya y al cual estará vinculado como presidente de su Consejo de Administración hasta el año 1972. Un año después recibiría el Premio Carlomagno por su labor en favor de la construcción de Europa.
\end{abstract}

PALABRAS CLAVE: Congreso de Europa; Cultura; Sociedad de Naciones; Colegio de Europa; Integración Europea.

\begin{abstract}
The idea of Europe in Salvador de Madariaga focused on the belief that without the previous creation of a European conscience, understood as the capacity to think the problems of Europe above the borders of the European states, any project of political or economic integration would be incomplete. Subjective solidarity, able to unite people beyond their material interests, must complete a de facto solidarity derived from the political and economic relations between the states. Hence, their problems will be common ones solvable through common plans. The key would be the existence of a European elite, endowed with a European conscience, able to provide a European soul to all the European institutions. This is the idea that Salvador de Madariaga supports in the Congress of Europe in The Hague (1948) and is put into practice in the College of Europe in Bruges (1949). The college, founded after the initiative of Madariaga, had him as the president of the board of directors up to 1972. One year later, the Charlemagne Prize was awarded to him for all his work in favor of the construction of Europe.
\end{abstract}

KEYWORDS: Congress of Europe; Culture; League of Nations; College of Europe; European Integration.

* Correspondencia a / Corresponding author: Víctor Gavín Munté. Sección de Historia Contemporánea y Mundo Actual. Departamento de Historia y Arqueología. Facultad de Geografía e Historia. Universidad de Barcelona. c/ Montalegre, 6-8 (08001 Barcelona-Spain) - -vgavin@ub.edu - https://orcid.org/0000-0003-0380-7152

Cómo citar / How to cite: Gavin, Víctor (2021). «El tiempo de Madariaga y la idea de Europa: Salvador de Madariaga en el Congreso de Europa en La Haya (1948)», Historia Contemporánea, 67, 705-733. (https://doi.org/10.1387/hc.22245).

Recibido/Received: 2020-11-16; Aceptado/Accepted: 2021-03-26.

ISSN 1130-2402 - eISSN 2340-0277 / (C) 2021 Historia Contemporánea (UPV/EHU) 
Europa no se hará de golpe ni en una construcción de conjunto: se hará mediante realizaciones concretas, creando primero una solidaridad de hecho.

Robert Schuman, Ministro Francés de Asuntos Exteriores, 9 de mayo de 1950

La solidaridad objetiva es material y pasiva, es un hecho, pero no crea nada (...) La solidaridad subjetiva es espiritual y activa; irradia, es creativa y apta para hacer surgir los sentimientos de cooperación y ayuda mutua. Sin esta forma de solidaridad que emana libremente del espíritu humano, la otra forma se vive de manera pasiva, no es otra cosa que una carga y una vergüenza.

\section{Salvador de Madariaga, L'Esprit de l'Europe, Mouvement Européen, 1952}

\section{El contexto: Estados Unidos y Europa}

La devastación que supuso la Segunda Guerra Mundial, tan solo dos décadas después de la Primera, tuvo una consecuencia prácticamente olvidada en la actualidad: el cuestionamiento de la organización política y económica de Europa basada en el estado-nación. Si nos trasladamos al final de los años 40 y principios de los años 50 del pasado siglo, los conceptos Europa y guerra iban de la mano. Europa era el continente de la Ilustración, pero era también el continente de las guerras sin fin, siendo los dos últimos conflictos unos que no se habían mantenido dentro de los confines de Europa, sino que habían alcanzado una dimensión mundial.

Tal estado de cosas había preocupado desde hacía siglos a multitud de pensadores europeos, quienes habían elaborado planes para conseguir una paz permanente en Europa. Sería el caso, por ejemplo, del filósofo Immanuel Kant ${ }^{1}$. Normalmente dichos planes coincidían en que había que cambiar la organización política del continente señalando a la estructura establecida como la causa de los conflictos. Tal estructura, a

${ }^{1}$ El texto del plan de Inmanuel Kant para una paz perpetua en: https://www.mtholyoke.edu/acad/intrel/kant/kant1 htm [página web consultada el 27 de octubre de 2020]. Una muy completa compilación de los diversos planes para organizar un gobierno europeo o mundial desde el siglo XIV es Wynner \& Lloyd (eds.), 1949. 
partir del siglo XVII, se basaba en el estado-nación soberano e independiente el cual además pretendía ser autosuficiente. Ello conllevaba una dinámica de rivalidad continua entre estados, que se traducía inevitablemente en guerras. No es de extrañar, por tanto, que los cambios que se sugerían señalaran a la necesidad de crear una nueva dinámica centrada en la identificación y gestión de intereses comunes como medio para evitar los conflictos. La puesta en práctica de tales ideas siempre chocaba con los intereses de los gobiernos quienes no mostraban ninguna disposición a abandonar el marco político que legitimaba su poder a favor de una estructura por encima de los estados que recortara su capacidad de decisión unilateral e independiente y creara una nueva legitimidad. A todo lo más, se llegaba a identificar algún interés puntual común que se traducía en una alianza también puntual pero que en ningún caso suponía la renuncia al ejercicio independiente del poder.

Frente a esta situación encontramos el proceso de construcción de los Estados Unidos el cual, con todos los problemas que encontró, guerra civil incluida, tuvo como resultado un tipo de organización política y económica completamente diferente a la de Europa. Un modelo federal, con una división simple, en el texto constitucional, del poder entre los estados y el gobierno federal, y un gigantesco mercado interior sin barreras fuente de su prosperidad. Consecuencia de ello encontramos, por una parte, la fascinación de europeos como Alexis de Tocqueville, Jean Monnet o Winston Churchill y, por otra, la voluntad de los Estados Unidos de mantenerse alejados de Europa y mantener a Europa lejos de América. No sería exagerado afirmar que para los Estados Unidos, una entidad política formada por europeos que habían emigrado de Europa, ya sea por motivos políticos, económicos o religiosos, la posibilidad de crear un nuevo estado en una tierra pretendidamente virgen, representaba la oportunidad de crear una especie de anti-Europa. El nuevo experimento político debía ser todo aquello que Europa no era y les había hecho cruzar el Atlántico en busca de mejor fortuna. No debe sorprender que la Farewell Address $(1796)^{2}$ de George Washington y la Monroe Doctrine $(1823)^{3}$ de James Monroe se convirtieran en los ejes de la política exterior de los Estados Unidos hasta el fin de la Segunda Guerra Mundial.

${ }^{2}$ La Farewell Address de George Washington en: http://avalon.law.yale.edu/18th century/washing.asp [página web consultada el 27 de octubre de 2020].

${ }_{3}$ El texto de la Doctrina Monroe en: http://avalon.law.yale.edu/19th_century/monroe. asp [página web consultada el 27 de octubre de 2020]. 
La situación en 1945 y durante los años previos no sería muy diferente a la aquí esbozada en el sentido de la existencia de grupos de europeos que señalaban a la organización basada en el estado-nación como la fuente de los conflictos y a la necesidad de superarla con un modelo federal $^{4}$. Sería este el caso del Conde Coundenhove-Kalergi y su idea de la Pan-Europa ${ }^{5}$.

También el de la británica Federal Unión que, en 1940 por ejemplo, publicó Uncommon Sense con el objeto de sensibilizar e informar a la opinión pública británica acerca de la necesidad de ir hacia una organización federal. En el mismo no se duda en afirmar que «the World is organised in the wrong way- in an out-of-date way». Sugiriendo en su lugar:

that nations (...) can submit their common interests to a common authority. For them it proposes a single army, navy and air force, a single tariff policy, a single money, a single postage system, and above all a central (federal) government. This would be elected by Tom, Dick and Harry, the citizens of the Union, not by their national governments. (...) Our federal government would deal with whatever concerned the whole union (e.g. tariffs) and with nothing else whatsoever. In every other respect the nations would keep, or indeed recover their independence and individuality. They would not be reduced to status of mere counties (...) The declared policy of the supporters of Federal Union in this country includes encouraging the setting up of permanent joint institutions with the USA and the USSR as the basis for complete union later, and at the same time the formation of an effective Federal movement on the Continent through propaganda here and abroad. ${ }^{6}$

Otro colectivo de defensa de la idea federal en Europa son los grupos de Resistencia al nazismo. En relación con el futuro político del continente europeo, el Manifiesto de Ventotene (1941), redactado por los italianos Altiero Spinelli y Ernesto Rossi durante su cautiverio en una prisión de la Italia fascista en la isla del mismo nombre, puede considerarse

${ }^{4}$ La major compilación de dichos planes continua siendo: Lipgens (ed.),1986.

5 Coudenhove-Kalergi, 1943; sobre el proyecto Pan-Europa ver ídem, 2010. Sobre los planes de creación de un gobierno o federación mundial, especialmente en los Estados Unidos, sigue siendo útil: Wooley, 1988, pp. 14-82.

${ }^{6}$ Uncommon Sense. A pamphlet for now. 1940. Consultable en la página web de The Federal Union: http://www.federalunion .org.uk/wp-content/uploads/2010/08/uncommonsense.pdf [página web consultada el 27 de octubre de 2020]. 
uno de los documentos fundacionales de dicho grupo. Sus palabras son claras:

«the question which must be resolved first, failing which progress is no more than mere appearance, is the definitive abolition of the division of Europe into national sovereign states» y se reclama la constitución de «a steady federal state, that will have at its disposal a European armed service instead of national armies, that will break decisively economic autarkies, the backbone of totalitarian regimes, that will have sufficient means to see that its deliberation for the maintenance of common order are executed in the individual federal states, while each state will retain the autonomy it needs for a plastic articulation and development of political life according to the particular characteristics of the various peoples». ${ }^{7}$

En los meses finales de la guerra en Europa, grupos de resistentes de diferentes nacionalidades se reunieron en diversas ocasiones en Ginebra, durante la primera mitad del año 1944, reuniones que culminaron en una Draft Declaration of the European resistance movements (20 May 1944) en la que se señala a Europa como la clave de la paz mundial, al estado nación pretendidamente autosuficiente como el culpable de la guerra y a la necesidad de crear una unión federal europea la cual:

must not interfere with the right of each of its member states to solve its special problems in conformity with its ethnical and cultural pattern. But in view of the failure of the League of Nations, the States must irrevocable surrender to the federation their sovereign rights in the sphere of defence, relations with powers outside the union, international exchange and communications (...) a government responsible not to the governments of the various member states but to the peoples, who must be under its direct jurisdiction in the spheres to which its powers extend. ${ }^{8}$

${ }^{7}$ El Manifiesto de Ventotene puede consultarse en la página web de la Union of European Federalists: http://www.federalists.eu/uef/library/books/the-ventotene-manifesto/ [página web consultada el 27 de octubre de 2020].

${ }^{8}$ La Draft Declaration of the European resistance movements (20 de mayo de 1944) puede consultarse en The European Navigator: https://www.cvce.eu/en/obj/draft_declaration_of_the_european_resistance_movements_20_may_1944-en-d68ca0ad-c24b-49068235-96b82814133a.html [página web consultada el 27 de octubre de 2020]. 
En resumen, lo que estos europeos están defendiendo no es la desaparición de los estados europeos sino la pérdida de su capacidad de acción unilateral en favor de una autoridad federal competente en aquellas áreas más susceptibles de provocar tensiones internacionales. Y un detalle muy importante es que esta autoridad federal debía derivar su poder no de los estados sino de los ciudadanos. Parece obvio que el modelo norteamericano debía estar en la mente de todos ellos.

Lo cierto es que, si nos referimos a estas personas como un movimiento político, debemos establecer que es un movimiento sin una base importante de ciudadanos que la apoye. Son básicamente grupos de intelectuales que elaboran planes para el futuro de Europa y del Mundo sin disponer de los resortes del poder. Los ciudadanos durante y tras la guerra están preocupados por su supervivencia y sus necesidades básicas no por las ideas federales, mientras las élites de gobierno europeas lo que buscan es la reconstitución de sus respectivos estados, de los cuales derivan su posición, con los cambios constitucionales necesarios para impedir una concentración de poder al estilo del partido Nazi en Alemania. Por lo tanto, a nivel europeo, podemos concluir que estamos ante una idea propia de unas élites intelectuales que no era compartida por las élites de gobierno.

Si cruzamos el Atlántico encontramos una diferencia importante. Aquí la idea acerca de la necesidad de modificar la organización política de Europa, era compartida por élites intelectuales y de gobierno. En el plano intelectual, destaca poderosamente la figura del periodista del New York Times y fundador de la Interdemocracy Federal Union, Clarence K. Streit, autor del libro Union Now. A proposal for a Federal union of the leading democracies (1940) ${ }^{9}$. La idea de Streit iba más allá del ámbito europeo y defendía la creación inicial de una unión federal de democracias del Atlántico norte: Estados unidos, el Reino Unido y la Commonwealth, Francia, Bélgica, Países Bajos, Suiza, Dinamarca, Noruega, Suecia y Finlandia. Sería una federación abierta al resto de democracias que federaría ciudadanía, defensa, aduanas, economía y comunicaciones. Este gobierno federal derivaría su legitimidad de los ciudadanos, no de los gobiernos. Para Streit la diferencia entre liga y unión era clara. Una liga es un gobierno de gobiernos para los gobiernos, una unión es un gobierno para el pueblo. En sus propias palabras: «a league is made for the state, a union

${ }^{9}$ Streit, 1940. 
is made for man» ${ }^{10}$. Más allá de las ideas de Streit, lo cierto es que en los Estados Unidos tuvo lugar un interesante debate intelectual acerca de la necesidad de trasladar el modelo organizativo de los Estados Unidos a Europa para acabar con los eternos conflictos del Viejo Continente. Ejemplos de ello serían el senador por Utah, Elbert Thomas, quien en su libro The Four Fears (1944), ataca los miedos de los Estados Unidos a las «entangling alliances», a Inglaterra, a Rusia y a la revolución para defender la superación de los marcos nacionales delimitados por fronteras y «share America's social, political, and economic security with the World»; por lo que a la legitimidad se refiere, Elbert Thomas no tiene dudas entre gobierno y ciudadanos: «sovereignty is we the people», escribe en su libro ${ }^{11}$. Otro ejemplo es el profesor Carl Van Doren quien en 1948 publica The great rehearsal. The story of the making and ratifying of the Constitution of the United States, donde señala que los enemigos de la constitución de los Estados Unidos en el siglo XVIII eran like the enemies of World federation in 1948, now when it is obvious that no dificulty in the way of a World government can match the danger of a World without it ${ }^{12}$. El contrapunto lo ofrecería el profesor emérito de historia en la Universidad de Cornell, Carl L. Becker, quien 1944 publica el libro How new will the better World be? A discusión of post-war reconstruction. En sus páginas el professor Becker indica:

it is quite futile to discuss post-war reconstruction on the assumption that the sentiment of nationalism will be any less strong than it has been, or the nations will cherish their sovereign independence any less than they have, or be less disposed to defend and promote their real or supposed national interests, or be less concerned with a balance of power that is advantageous to them. ${ }^{13}$

En el área de la acción política los senadores Elbert Thomas y el senador por Arkansas, William Fulbright, presentaron, el 21 de marzo de 1947 ante el Senado, la propuesta de resolución numero 10 (el representante por Louisiana, Hale Boggs, haría lo mismo ante la Cámara de Representantes, tres días después) la cual señalaba que «The Congress favors the

\footnotetext{
10 Ídem, p. 9.

11 Thomas, 1944, pp. 155 y 171.

12 Van Doren, 1948, p. X.

13 Becker, 1944, pp. V y VI.
} 
creation of a United States of Europe within the framework of the United Nations» ${ }^{14}$. La resolución no tuvo una gran acogida en las cámaras, aunque sí fue recibida con interés por la prensa ${ }^{15}$. Lo cierto es que, aun y con la fría acogida política, la dimensión mundial de los dos últimos conflictos, originados en Europa pero que habían arrastrado a los Estados Unidos, demostraría que la política basada en los «no foreign entanglements» defendida desde los tiempos de George Washington y Thomas Jefferson ya no era útil para garantizar la paz y la prosperidad de los Estados Unidos ${ }^{16}$. Es a partir de tal asunción que el gobierno norteamericano, legislativo y ejecutivo, entenderá que la organización de Europa era un asunto de su incumbencia. Nadie se expresó con mayor claridad a tal efecto que el entonces Senador y futuro Secretario de Estado, John Foster Dulles, en 1948:

Twice within the last twenty-five years the United States has become deeply involved in the wars originating between the independent, unconnected sovereignties of Europe. It has been demonstrated that the world has so shrunk that European wars can no longer, as during the last century, be confined to Europe. Therefore, it is not merely of selfinterest to Europe, but of vital concern to us, that there be not restored in Europe the conditions which inherently give rise to such wars. From a purely selfish standpoint any American program for peace must include a federated continental Europe. ${ }^{17}$

${ }^{14}$ Farnetti, 2004, p. 117. Sobre el Senador Fulbright y la integración de Europa ver: Woods, 1995, pp. 137-144.

15 Un ejemplo de las dudas que la superación del marco del estado-nación europeo levantaba entre el gobierno de los Estados Unidos es la opinión del Secretario de Estado George C. Marshall sobre la Resolución Fulbright-Thomas. Respondiendo al senador republicano Arthur Vandenberg sobre la cuestión a una semana de pronunciar su famoso discurso en la Universidad de Harvard que lanzaría el plan que llevará su nombre, indicó: «I am deeply sympathetic toward the general objective (...) but the United States had to make it clear that it did not intend to controvert the hallowed principle of national self-determination», Woods, 1995, p. 142.

${ }^{16}$ Fue Thomas Jefferson quien defendió, honest friendship with all nations, entangling alliances with none en el discurso inaugural de su presidencia el 4 de Marzo de 1801. Consultable en: http://avalon.law.yale.edu/19th_century/jefinau1.asp [página web consultada el 27 de Octubre de 2020]; Por su parte George Washington aconsejó a sus conciudadanos en su Farewell Address en 1796: It is our true policy to steer clear of permanent alliances with any portion of the foreign world. Consultable en: http://avalon.law.yale.edu/18th_century/washing .asp [página web consultada el 27 de octubre de 2020].

17 The Unification of Western Europe (4 June 1948) John Foster Dulles Papers Collection, Seeley G. Mudd Manuscript Library, Princeton University. 
Foster Dulles aludía a la necesidad de cambiar la organización de Europa para evitar nuevas guerras originadas en territorio europeo, pero evitar guerras no sería el único objetivo. Europa, devastada por el último conflicto, tenía unas necesidades colosales para su reconstrucción. Reconstrucción que se convirtió en un objetivo político de primer orden a partir del inicio de la Guerra Fría, al valorarse la puesta en funcionamiento de la economía europea y la solución de las necesidades más básicas de los europeos como el mejor antídoto ante la expansión del comunismo. Ante ello, el gobierno de los Estados Unidos concluyó que Europa necesitaba obtener el máximo rendimiento de sus recursos a partir de una gestión eficiente de los mismos lo cual requeriría una integración de las economías europeas para crear un mercado único comparable al norteamericano. Aquí quien se expresó con suma claridad fue el futuro presidente de los Estados Unidos Dwight Eisenhower:

It is scarcely necessary to enumerate the problems that arise out of or are exaggerated by the division of West Europe into so many sovereign nations. Norway is short of manpower, Italy way over. Italy has excess productive capacity in vehicles and planes-many others have none at all! France and Germany (the key powers of the region) are on opposite sides in many problems because of French hatred for the Boche as well as the fear of a restored Western Germany. Each nation watches its neighbor to see that it's neighbor's contribution to the common security is at least equal to the first nation ratio- and none is ever so convinced! (...) I think that the real and bitter problems of today would instantly come within the limits of capabilities in solving them if we had this single government! Moreover, I believe inspired leaders could put it across. But everyone is too cautious, too lazy and too ambitious (personally). So many advantages would flow from such a union that it is a tragedy for the whole human race that it is not done at once. (...) With this one problem solved-all lesser ones could soon disappear. I could write a volume on the subject.

El futuro presidente de los Estados Unidos también incidía en que la puesta en práctica de tales ideas no debía suponer la desaparición de los estados nación ni tan sólo una uniformización de las formas políticas: Local governments would not necessarily be identical. It would be necessary that each adopt and observe a simple «bill of rights». Socialist Sweden would live alongside a capitalist Germany, but with the elimination of trade barriers and all economic and political restraint on free move- 
ments ${ }^{18}$. El tono utilizado por Eisenhower denota su enojo ante la inacción de los europeos para con algo que para él era una necesidad evidente, pero el gobierno de los Estados Unidos, a diferencia de los federalistas europeos, creía disponer de una herramienta que forzaría a los gobiernos del continente a integrar sus economías e iniciar la vía federal: la ayuda financiera y material norteamericana para la recuperación de Europa y vehiculada a través del Plan Marshall. El Plan estableció desde su inicio que su objetivo no era ayudar a la recuperación de una docena de economías autosuficientes sino a la economía europea en un su conjunto para lo cual la integración era imprescindible. Lo cierto es que con el Plan Marshall se inició una actitud de los gobiernos europeos para con los Estados Unidos que llegaría hasta la derrota la Comunidad Europea de Defensa en agosto de 1954: decir a Washington que estaban de acuerdo con sus objetivos políticos para reformar Europa, con el fin de no perder la ayuda que los Estados Unidos estaban dispuestos a ofrecer a tal efecto, cuando ello no les interesaba en absoluto. A ello se refería el principal administrador del Plan Marshall, Paul G. Hoffman, cuando el 31 de octubre de 1949 les recordó que la integración «is a vital objective. It was to this that Secretary Marshall pointed in the speech which sparked Europe to new hope and new endeavour. It was on this promise that the Congress of the United States enacted the Economic Cooperation Administration Act. This goal is embedded in the Convention of the OEEC $\gg^{19}$. Consecuencia directa de esta advertencia es el mensaje que Ernest Bevin, Secretario del Foreign Office, envió a su colega norteamericano. Entendiendo que el objetivo político buscado y esperado por Washington no era negociable, Londres decidía quedarse al margen de la construcción de la Europa federal, dando inicio a una actitud que, con altibajos, llega hasta nuestros días:

Proposals may now be put forward which if they were to be accepted by His Majesty's Government might involve some degree of merging or integration of the United Kingdom economy with the economies of Western European countries. Although no actual proposals have been formulated, or at least communicated to the United Kingdom,

18 Galambos (ed.), 1989, pp. 340-341.

19 El texto del discurso de Paul G. Hoffman el 31 de Octubre de 1949 puede consultarse en The European Navigator, CVCE de la Universidad de Luxemburgo: https://www. cvce.eu/content/publication/2009/4/3/840d9b55-4d17-4c33-8b09-7ea547b85b40/publishable_en.pdf [página web consultada el 27 de octubre de 2020]. 
we have considered the general principles involved. We must have regard to the position of the United Kingdom as a power with worldwide responsibilities for administration and defence and as the leading member of the British Commonwealth and sterling area as well as to the general responsibilities which we have assumed under the North Atlantic Pact, and the similar obligations under the Brussels Treaty. (...) In summary, the principal objective of our policy is to reconcile our position as a world power, as a member of the British Commonwealth, and as a member of the European community. We believe that we can effect this reconciliation but that if we are to do so, we cannot accept obligations in relations to Western Europe which would prevent or restrict the implementation of our responsibilities elsewhere..$^{20}$

La situación parecía clara. La idea de federalizar a Europa era compartida por intelectuales a ambas orillas del Atlántico. La política norteamericana, por su parte, no dudaba ya de la necesidad de reformar las tradicionales estructuras políticas y económicas del Viejo Continente. Estaríamos, por tanto, ante una idea transnacional. El problema era que su puesta en práctica dependía de que la misma convicción fuera compartida por los gobiernos y los parlamentos de Europa. Y aquí esta convicción no existía.

Robert Schuman, ministro francés de Asuntos Exteriores, ha pasado la historia como uno de los padres fundadores de Europa, lo cual se debe, esencialmente, a la relación con el lanzamiento del plan que lleva su nombre, pero sería un error querer ver en ello una identificación con el federalismo. A finales de 1948, cuando se estaba debatiendo la creación del Consejo de Europa, Schuman no duda en alinearse con los Británicos, respecto del limitado poder que dicha organización debía poseer, y en calificar a aquellos franceses que defienden unos más amplios poderes para la primera institución europea como «wilder federalist colleagues» que conseguirán apartar al Reino Unido de la Europa continental ${ }^{21}$.

20 «Personal Message to the Secretary of State from Mr. Bevin, dated 25th October 1949», Foreign Relations of the United States (FRUS) 1949, Vol. 4, pp. 347-48. Este mensaje seguía a una confidencia del British Chancellor of the Exchequer, Sir Stafford Cripps, al periodista norteamericano Walter Lippman en marzo del mismo año: «that he would much rather go it alone, and not be obligated to make agreements with the other European nations.» Para Lippman ello suponía «a major defeat of a declared American purpose.» Ver Woods, 1995, p. 143.

${ }^{21}$ Kaplan, 1984, p. 92. 


\section{Salvador de Madariaga}

En este contexto de cuestionamiento del tradicional orden internacional europeo e incluso mundial, ¿cuál fue el papel de Salvador de Madariaga)? ¿Hasta qué punto sus ideas respecto del futuro de Europa y del Mundo coincidían con las de los personajes hasta aquí mencionados? Si hubiera sido el Ministro de Asuntos Exteriores de una república o una monarquía parlamentaria españolas en la postguerra mundial, ¿se hubiera identificado con proyectos como la Comunidad Europea del Carbón y del Acero (CECA), la Comunidad Europa de Defensa (CED) o la Comunidad Económica Europea (CEE)? $?^{22}$

Si hemos de definir a la persona de Salvador de Madariaga (de ahora en adelante SdM), nacido en La Coruña en 1886 y fallecido en Locarno el 14 de diciembre de 1978, lo más sensato es empezar por su propia autodefinición: yo soy un trabajador intelectual ${ }^{23}$. Hijo de una familia acomodada, educado en Francia, ingeniero de formación, intelectual de vocación, político por responsabilidad y lealtad institucional. No debe sorprender, por tanto, que en el obituario que le dedica The Washington Post, se le defina primero como «eminent liberal Spanish writer», en segundo lugar, como «historian» y sólo en último término como «former diplomat $»^{24}$. Y a quien esto suscribe le parece que el propio SdM hubiera estado perfectamente de acuerdo tanto con la descripción como con el orden de la misma.

Si consultamos una de las fuentes de referencia en red sobre la historia de la integración europea, The European Navigator, encontramos una detallada información sobre su trayectoria: Director de la Sección de Desarme del Secretariado de la Sociedad de Naciones (1921-1928); Profesor de Literatura Española en la Universidad de Oxford (1928-1932); Embajador de España en Washington (1931) y París (1932); Ministro de Instrucción Pública y de Justicia (1934); Representante de España en la Sociedad

${ }^{22}$ Sobre la relación entre Salvador de Madariaga y el proyecto europeo que llega hasta nuestros días es interesante el análisis de Sobrino, 2003, pp. 759-775.

${ }^{23}$ Salvador de Madariaga: «Auto-entrevista», en $A B C, 28$ de noviembre de 1971, p. 134 [consultada en la Hemeroteca on-line de $A B C$, https://www.abc.es/archivo/periodicos/abc-madrid-19711128-134.html, el 27 de octubre de 2020].

24 «Salvador de Madariaga, Writer, ExDiplomat, Dies», en The Washington Post, 15 de diciembre de 1978 [consultada en la Hemeroteca on-line de The Washington Post, https://www.washingtonpost.com/archive/local/1978/12/15/salvador-de-madariaga-writerex-diplomat-dies/bc1012f7-4f10-4278-ad9a-52fb7761c81b/ [el 27 de octubre de 2020]. 
de Naciones (1935-1936); Profesor y Escritor (1928-1978); Presidente del Consejo Administrativo del Colegio de Europa en Brujas (1950-1972); Premio Carlomagno (1973) 25 . En resumen, 13 años de política activa ya sea representando a España en la Sociedad de Naciones, en embajadas o ejerciendo de ministro, frente a medio siglo de actividad intelectual. Nadie puede discutir la precisión de la autodefinición de SdM como trabajador intelectual apuntada más arriba. Otra cosa es la dimensión y contenido políticos de buena parte de su trabajo en las aulas y fuera de ellas.

No estamos por tanto ante un político al uso, con una formación pensada para una vida de actividad gubernamental o parlamentaria, a la vida de partido y apta para el ejercicio del poder político en sus diversas formas. A ello cabe añadir que, a su falta de vocación para tal objetivo, el contexto nacional tampoco le ayudó. Como español adulto le tocó convivir con la Monarquía de Alfonso XIII (1902-1931) la Segunda República (1931-1939), sistema para el que trabajó pero con el que nunca se identificó completamente por su falta de orden; y el Franquismo (1939-1975) al que siempre atacó por su falta de libertades ${ }^{26}$. En la auto-entrevista anteriormente citada, $\mathrm{SdM}$ no duda en señalar que tiene una sola pasión: la pasión por la libertad personal a la vez que se define como conservador porqué estimo que sin un mínimo de orden no puede haber ni libertad ni justicia. Y es que como también indica, la democracia es una cuestión de formas y maneras, mientras que la libertad es una esencia y un fin ${ }^{27}$.

Tampoco le acompañó la suerte en la vida internacional. Su etapa como representante de España en la Sociedad de Naciones, en el llamado periodo de entreguerras, le provocó más sinsabores que satisfacciones. A ser miembro de una organización desprovista de cualquier poder real re-

25 «Salvador de Madariaga», en The European Navigator: https://www.cvce.eu/en/obj/ salvador_de_madariaga-en-3a5e8b92-e3c3-43ba-965f-71b156696110.html [página web consultada el 27 de octubre de 2020].

26 de Madariaga, 1959.

27 Salvador de Madariga: «Auto-entrevista», en $A B C, 28$ de noviembre de 1971, p. 139 [op. cit. consultada en la Hemeroteca on-line de $A B C$ el 27 de octubre de 2020]. Cabe citar también las palabras que sobre SdM escribió el Catedrático de Historia Moderna de la Universidad Autónoma de Barcelona, Ricardo García Cárcel: Un español avanzado a su tiempo y desubicado (...) Un español distinto. Un español de una España que nunca fue, que nunca pudo ser, en «Memoria de Salvador de Madariaga», $A B C, 14$ de diciembre de 2003 [consultada en la Hemeroteca on-line de $A B C$ : https://www.abc.es/archivo/periodicos/abc-madrid-20031214-3.html, el 27 de octubre de 2020]; ver también el «Estudio introductorio» de Beneyto en: de Madariaga, 2010, pp. 5-33; Preston, 1999, pp. 193-226. 
levante para evitar una nueva guerra mundial, se le unía la falta de apoyo desde Madrid. Su posición en favor de China y en contra de Japón durante la crisis de Manchuria motivó el rechazo tanto de Luis de Zulueta, Ministro de Asuntos Exteriores, como de Manuel Azaña, Presidente del Consejo de Ministros, quienes le acusaban de lanzarse a quijotadas contrarias al interés de España ${ }^{28}$. Quizá su mayor logro en este período se reflejó en la trasposición del Pacto Kellogg-Briand de 1928, por el cual los estados firmantes renunciaban a la guerra como instrumento de la política nacional en las relaciones entre ellos, en el artículo 6 de la Constitución Española de $1931^{29}$. Aunque viendo la evolución de tal concepto en España, Europa y el Mundo en los años venideros, difícilmente SdM acabaría considerando dicha inclusión como un éxito.

La Guerra Civil Española (1936-1939) provocará el exilio de SdM y no regresará a España hasta después de la desaparición del General Francisco Franco en 1975, aunque intentará, sin éxito, promover una intervención británica para detener la misma a través de su amigo y colega en la Sociedad de Naciones, Anthony Eden, en los primeros meses de la contienda $^{30}$. La Segunda Guerra Mundial (1939-1945) sólo hará que reafirmar la nula confianza de SdM en el orden internacional de los años 30 del que él había formado parte.

\section{De la postguerra a La Haya}

Las convicciones de SdM son profundas y los hechos que le ha tocado vivir hasta ahora no hacen otra cosa que reafirmarlas. El valor supremo es la libertad individual la cual está por encima de naciones y fronteras. En palabras de SdM: nous n'accordons à personne d'autre, et moins qu'à personne, à l'Etat, le droit ou la compétence de nous sauver de l'erreur au prix de notre liberté. Ce n'est donc pas seulement notre fierté qui nous force d'être libres ; c'est aussi notre sens commun; car nous savons que les erreurs de la liberté, c'est para davantage de liberté qu'elles se corri-

${ }_{28}$ Paul Preston: «Don Quijote de la Manchuria», en $A B C, 30$ de julio de 1995, p. 3 [consultada en la Hemeroteca on-line de $A B C$ : https://www.abc.es/archivo/periodicos/abcmadrid-19950730-3.html, el 27 de octubre de 2020].

${ }_{29}$ Tamayo Barrena, 1984, pp. 187-213, ver especialmente la nota al pie $n .^{\circ} 99$ en las páginas 212-213.

${ }^{30}$ Ver de Madariaga, 1983, pp. 229-257, y Pazos, 2009, pp. 317-332. 
gent $^{31}$. El lugar de nacimiento es algo que marca el carácter del individuo durante toda su vida pero que en ningún caso debe limitarle ni su libertad de acción ni sus miras ${ }^{32}$. Los estados-nación son indispensables, pero no pueden monopolizar la soberanía. En este caso SdM siempre mira hacia arriba, hacia el Mundo, más allá de Europa, pero nunca hacia abajo. El nacionalismo catalán, vasco o gallego son meros provincianismos limitadores. La libertad sin orden no existe, y por ello el sistema político sólo es válido si garantiza ambos ${ }^{33}$. Relacionado con el concepto de orden, está el concepto de jerarquía. SdM cree en la jerarquía social y en la indispensable capacidad de dirección de una élite culta y educada resultado de una formación acorde con sus responsabilidades, principio que expresa en un discurso que pronuncia en la Universidad de La Sorbona en París durante el cual expresa incluso simpatías hacia el fascismo italiano:

(...) las clases directoras de Europa se revelan inferiores a la labor que habían heredado del pasado, con lo cual los estados modernos se ven inevitablemente abocados a una especie de nivelación social por derrumbamiento de su jerarquía. Es de justicia reconocer que debemos al fascismo italiano el servicio de haber puesto de relieve desde sus comienzos este síntoma de nuestras democracias enfermas. Mientras que un historiador francés muy siglo XIX decía: «No hay minoría selecta, son meras palabras presuntuosas», el fascismo italiano titulaba «Jerarquía» la revista de sus teorizantes (...) Los ensayos de constitución corporativa del estado que, con circunspección y perseverancia admirables, viene haciendo el Duce, merecen la atención más sostenida de los verdaderos demócratas. Tratase de un organicismo si se permite la palabreja, de buena ley. ${ }^{34}$

31 Discours de Salvador de Madariaga à la conférence européenne de la culture (Lausanne, 8 décembre 1949) en https://www.cvce.eu/obj/discours_de_salvador_de_ madariaga_a_la_conference_europeenne_de_la_culture_lausanne_8_decembre_1949-frafc2551e-6fd4-433f-8315-888764c5f1e9.html [página web visitada el 27 de octubre de 2020].

${ }^{32}$ Su obra más conocida, Bosquejo de Europa, 1951 es un verdadero canto a la diversidad de Europa y los europeos que ningún proyecto de integración debe menoscabar.

${ }^{33}$ En tal principio se asienta su obra más polémica, Anarquía o jerarquía. Ideario para la constitución de la tercera república, 1935, de la cual regalará un ejemplar al General Franco en una comida entre ambos celebrada antes del Alzamiento de Julio de 1936, ver González Cuevas, 1989, pp. 145-181 (cita en la página 167).

34 «Discurso pronunciado en la Sorbona en ocasión de la apertura del Curso del Centro de Estudios de la Revolución francesa», en París el 25 de noviembre de 1933: en de Madariaga, 1934, pp. 83-110 (citas en las páginas 99-100). Resulta interesante analizar el 
Esta defensa de la labor necesaria de las élites otorga toda lógica al hecho que nuestro personaje acabe dirigiendo el Colegio de Europa en Brujas del cual será uno de sus impulsores. Se trata de un centro para la formación de una élite europea en una mentalidad europea por encima de las formaciones nacionales. Cuna de muchos altos funcionarios de las instituciones comunitarias. En este punto, las ideas de SdM encajarían perfectamente con el proyecto europeo iniciado en los años 50 del pasado siglo. No debe olvidarse que este ha sido desde su inicio un proyecto de arriba hacia abajo y nunca a la inversa. Las élites gubernamentales han sido las encargadas de dar forma y aplicar el proyecto sobre los ciudadanos de los estados-miembro tanto para garantizar el bienestar de aquellos como el progreso del estado. Cabe recordar que las primeras elecciones democráticas para un parlamento europeo con competencias muy limitadas no llegarían hasta 1979, casi tres décadas después de la Declaración Schuman de 9 de mayo de 1950. Y en relación con Europa, para SdM, esta es esencialmente un conjunto cultural y de valores compartimentados en diversos estados-nación. Es por ello que cualquier proyecto práctico de integración carece de sentido sino viene precedido de la superación de tales compartimentos para crear una verdadera conciencia europea. Incluso así, al ser el individuo libre el verdadero eje del pensamiento de SdM, sólo el Mundo en toda su amplitud podía ser el marco para el ejercicio pleno de dicha libertad. El que ello fuera un proyecto irrealizable por excesivamente ambicioso, creó en SdM una tensión permanente entre proyectos mundialistas y europeístas ${ }^{35}$.

Dicha tensión se reflejó en la primera publicación relevante de $\mathrm{SdM}$ tras la Segunda Guerra Mundial: ;Ojo, Vencedores! ${ }^{36}$ En ella conviven la

pensamiento de Salvador de Madariaga en el contexto del pensamiento español de la época especialmente la conexión entre modernización, europeización y democracia. Realizar dicho análisis en el marco de este artículo superaría en exceso la extensión permitida y su enfoque, pero pueden citarse algunos trabajos generales y concretos para el lector interesado. En cuanto a textos de carácter general ver: Beneyto, 1999; Pérez Garzón, «Modernización y europeización en el pensamiento español de la segunda mitad del siglo xx: hacia el fin de las angustias regeneracionistas», en Gómez Benito (coord.), 2011, pp. 199-237. En un plano más concreto de comparación con un pensador español de la misma generación que Salvador de Madariaga, la mejor opción es José Ortega y Gasset. En este caso ver: La rebelión de las masas y otros ensayos, 2014 y Meditación de Europa y otros ensayos, 2015.

35 Una excelente visión sobre la doble vertiente mundial y europea de Salvador de Madariaga es: Lago y Domínguez Castro 2017.

36 De Madariaga, 1945. 
idea de organización mundial y la idea de Europa. La diferencia estriba en que mientras de la primera escribe en un sentido fundamentalmente práctico, de la segunda lo hace en términos de conciencia. La primera hay que organizarla, la segunda hay que comprenderla ${ }^{37}$. El Comundo o colectividad mundial debería ser impulsada por la Organización de las Naciones Unidas, garantizar la libertad del individuo y resto de libertades fundamentales y supervisar la acción de los diferentes estados de acuerdo con un conjunto de valores compartidos ${ }^{38}$. La idea de la existencia de un conjunto de valores universales, compartidos por toda la humanidad, es uno de los puntos débiles en los sueños mundialistas de SdM. Fácilmente se deduce que los valores que considera universales son, esencialmente, los valores de Occidente ${ }^{39}$. Una concepción que encajará mal con el proceso de descolonización que iba a iniciarse a corto plazo y que, parece claro, nuestro autor nunca toma en consideración.

Europa no puede construirse sin una conciencia europea y el problema es que el centro cultural de Europa es demasiado débil ${ }^{40}$. Sin duda recordando todas las carencias de la Sociedad de Naciones de la que formó parte, SdM señala que de poco servirán comités, ligas, uniones o cualquier organización de estados europeos si falta pensar y sentir en europeo. La solución podría ser, siguiendo sus palabras, la creación de una Junta Europea que no fuera nacional ni internacional, formada por europeos que analizaran los problemas del continente como si no existieran las fronteras nacionales, sólo desde el punto de vista de la nación llamada Europa ${ }^{41}$. Aquí está prefigurando la misión del Colegio de Europa en Brujas, que se fundará 5 años después: formar a estos europeos con una visión europea de Europa. Y es que para SdM sólo la existencia de tal conciencia europea puede llenar de contenido real cualquier proyecto integrador por mucho que se utilicen todas las entradas de los diccionarios referentes a las uniones de naciones para etiquetarlos.

37 Ídem, p. 195.

38 Ídem, pp. 218-19.

39 Je veux bien que, dans un certain sens plus objectif et plus académique du mot, il n'y a pas de culture européenne qui ne soit aussi occidentale, voire universelle, en Discours de Salvador de Madariaga à la conférence européenne de la culture (Lausanne, 8 décembre 1949), op. cit.

40 de Madariaga, 1945, p. 199.

41 Ídem, p. 203. 
Es por todo ello que se hace difícil pensar que los proyectos tendentes a superar el tradicional orden internacional europeo, basado en la sacrosanta soberanía nacional de los estados-nación, elaborados durante la segunda mitad de los años 40 y hasta la Declaración Schuman del 9 de mayo de 1950 cumplieran la condición básica que SdM consideraba indispensable: la creación de un espíritu europeo entre los europeos que les hiciera mirar, comprender y apreciar por encima de las fronteras nacionales. El Plan Marshall, la OEEC (Organización Europea de Cooperación Económica), el Benelux, el Pacto de Bruselas, el Consejo de Europa, la OTAN (Organización del Tratado del Atlántico Norte, el Plan Schuman que daría lugar a la CECA (Comunidad Europea del Carbón y del Acero), son todos ellos proyectos multilaterales para facilitar la cooperación entre los estados y su progreso, sólo la CECA supondrá una pérdida real de soberanía exclusiva estatal en favor de un órgano supranacional en un sector económico muy concreto. Todos ellos tenían el potencial de crear una conciencia común, pero lo que para estos suponía el corolario del éxito del proyecto, para SdM resultaba ser la premisa indispensable de partida para tener éxito de verdad más allá de cifras y balances.

Al tratarse de un político en el exilio sin ninguna función de representación de ninguno de los estados participantes en los proyectos mencionados, $\mathrm{SdM}$ no tomó parte en el proceso de creación de ninguno de ellos. Sólo hubo un caso en que tal condición no fue indispensable, ya que ni la iniciativa fue estatal ni las deliberaciones o las conclusiones afectaban de manera obligatoria a ningún estado: el Congreso de Europa celebrado en La Haya en 1948 que daría lugar al Consejo de Europa un año después ${ }^{42}$. Lo cierto, es que una reunión, en su sentido más amplio, de partidarios de la unidad europea para debatir sobre dicha unidad, los desafíos que su realización planteaba y ofrecer soluciones a los gobiernos interesados, parecía un escenario más adecuado para alguien preocupado por el espíritu eu-

${ }^{42}$ Sobre el Congreso de Europa celebrado en La Haya los días 7-10 de mayo de 1948 ver el conjunto de recursos que ofrece The European Navigator (www.cvce.eu) [página web consultada el 27 de Octubre de 2020] El Congreso de Europa fue organizado por el Comité Internacional de Movimientos para la Unidad Europea (ICMEU) creado el 11 de noviembre de 1947 en París, incluyendo a la Unión Europea de Federalistas (UEF) de Henry Brugmans, el Movimiento para la Unidad Europea de Winston Churchill, la Liga Independiente para la Cooperación Europea (ILEC) de Paul Van Zeeland, el Consejo Francés por una Europa Unida de Raoul Dautry, los Nuevos Equipos Internacionales (NEI) de Robert Bichet y, desde Abril de 1948, la Unión Parlamentaria Europea (EPU) del Conde Richard Coudenhove-Kalergi. 
ropeo de los europeos que la Alta Autoridad de la CECA, los diferentes órganos del Plan Marshall o el Consejo Atlántico.

Aun y con ello, SdM, siempre fue consciente que la importancia de la reunión dependería de sus consecuencias posteriores, las cuales, a su vez, dependían de los gobiernos de los estados. En otras palabras, siempre entendió las limitaciones del evento más allá de la importancia y fama de sus asistentes y el patrocinio de la Casa Real de Holanda. El congreso dividió sus trabajos en 3 comités: político, económico y cultural, otorgando la organización la presidencia del tercero a $\mathrm{SdM}$, tras la renuncia del italiano Ignazio Silone. Y poca duda cabe que la cultura era el ámbito que mejor encajaba con alguien que, como hemos visto, años después se definiría como un trabajador intelectual.

La documentación del Congreso de Europa que se conserva en el Archivo Histórico de las Comunidades Europeas en Florencia nos permite examinar con detalle la actividad de SdM al frente del comité cultural del Congreso de Europa durante los días 7-10 de mayo de 1948. La primera intervención tiene lugar en la mañana del día 8 y SdM abre la sesión estableciendo con suma claridad porqué los reunidos están allí:

Nous sommes ici parce que nous voulons y être. Personne ne nous a forcés à venir ici. Tous ceux qui assistent à ce Congrès, du fait qu'ils y participent, affirment par là-même l'existence de quelque chose « sui generis » dans le monde qui s'appelle «l'Europe » (...) c'est parce qu'il me semble que du moment que nous sommes ici, nous croyons à l'Europe. ${ }^{43}$

Lo están por voluntad propia, no por mandato o en representación de nadie y porqué creen en Europa con lo cual si hay algo que no requiere debate es si Europa existe o no.

Seguidamente aparece el SdM más pragmático ofreciendo, por si fuera necesario, un baño de realismo a sus colegas de comité. ¿Qué somos? Nous ne sommes pas une Assemblée parlementaire; nous sommes un Assemblée d'amis qui voulons chercher la voie vers l'Union euro-

43 Congress of Europe, The Hague. Cultural Committee, Saturday, May 8th 1948, 10.15am Mr. Madariaga in the chair. Sessions of Committee-Consideration of Economic, Political and Cultural reports. ME-502 Actas de la Comisión Cultural en La Haya, Congreso de La Haya, Archivos Históricos de la Unión Europea, Florencia [de ahora en adelante, AHUE), p. 1. 
péenne. Con lo cual, señala, tenemos libertad pero no tenemos poder, es decir, nos votes n'ont pas une très grande importance $e^{44}$.

Durante esta primera sesión el trabajo de SdM consiste esencialmente en conseguir que el resto de participantes sean conscientes de donde están, para qué y con qué. Que debatan en profundidad y con toda libertad, pero sin pretender tener un poder que no tienen. SdM parece no albergar ninguna duda sobre el valor simbólico del Congreso, pero a la vez, del nulo poder del que gozan. Así, por ejemplo, cuando el debate se centra sobre la necesidad de crear instituciones que encarnen a Europa, SdM, sin negar la necesidad de las mismas, recuerda que para un congreso de orden privado y sin medios financieros significativos ello queda completamente fuera de su alcance. A lo más, advierte, podrá crearse un comité que se ocupe de trabajar con las conclusiones que se alcancen y tratar de influir sobre los gobiernos. Si algún asistente al Congreso de Europa cree estar presente en la creación de Europa, este no es Salvador de Madariaga.

Es al día siguiente, domingo 9 de mayo, curiosamente dos años antes del día en que se leerá la Declaración Schuman en París, el evento considerado como fundacional de la actual Unión Europa y celebrado como Día de Europa en toda la Unión, que, en sesión plenaria, SdM dejará ver a todos los delegados su opinión sobre Europa y los problemas que hay que superar. La cultura y el espíritu son la clave maestra de toda construcción europea, hasta el punto que sin ella los aspectos políticos y económicos se hunden. El problema de Europa es la divergencia entre la solidaridad de los hechos o solidaridad objetiva, y la solidaridad de los sentimientos o solidaridad subjetiva. La primera ya existe, guste o no, pero la segunda brilla por su ausencia. SdM utiliza una curiosa y efectiva metáfora. Es como el conductor de un coche en una caravana de vehículos. Los hechos le fuerzan a ser solidario con los demás automóviles, pero sus sentimientos los envían a todos al infierno. De la misma manera, no puede olvidar su experiencia en tal sentido en la Sociedad de Naciones durante los años 30:

Un souvenir de Genève éclairera d'un jour plus vif ce paradoxe, au fond, simple. Deux cas fameux d'agression international donnèrent lieu

${ }^{44}$ Congress of Europe, The Hague. Cultural Committee, Saturday, May 8th 1948, 10.15am Mr. Madariaga in the chair. Sessions of Committee-Consideration of Economic, Political and Cultural reports. ME-502 Actas de la Comisión Cultural en La Haya, Congreso de La Haya, AHUE, p. 3. 
à l'application de l'article 16 du Pacte de la Société des Nations, celui qui prévoyait des mesures contre l'Etat agresseur. Ces mesures, généralement connues sous le nom de sanctions dans les deux cas. L'étude précise des motifs de cet échec est des plus instructives. Disons « motifs » au pluriel, car il en est deux. Le premier c'était que les liens d'intérêts matériels entre les pays étaient trop enchevêtrés et trop denses pour permettre la rupture sans punir les punisseurs autant, ou même davantage, que les punis. Le second c'était que les torts infligés aux pays victimes n'avaient pas inspiré dans l'opinion publique de l'ensemble des pays un sympathie suffisante pour éveiller en eux la solidarité morale que la solidarité juridique du Pacte présupposait. Qu'est-ce à dire, si ce n'est que l'idée même des sanctions se trouvait donc isolée dans un terrain neutre entre la solidarité matérielle des peuples qui l'avait dépassée, et leur solidarité morale, qui restée retardataire ? C'est dans une situation analogue que nous nous trouvons quant à l'évolution de l'unité européenne. ${ }^{45}$

¿Cuál es el remedio para que la solidaridad subjetiva se ponga a la par con la objetiva?:

Il manque la prise de conscience de l'unité du continent. Ce qui manque à l'Europe, ce ne sont pas des attributions politiques et économiques, ce qui manque à l'Europe, c'est qu'elle n'existe pas là où un pays existe : un pays n'existe que dans le cœur de ces citoyens. Tant que l'Europe n'aura pas commencé à exister dans le cœur des citoyens, ce pays n'existe pas, il est inutile qu'il donne des institutions. ${ }^{46}$

Europa está compartimentada en estados pero ello no ha impedido las relaciones, a menudo trágicas, entre los habitantes de cada compartimento y ello debido a una doble tradición común: la socrática y la cristiana. La primera relacionada con la duda y el cuestionamiento constante y la segunda con la persona humana y el humanismo. Por tanto, ¿cuál es el cometido de los asistentes al congreso? (...) de travailler ensemble à l'Europe, et de faire qu'à force de travailler ensemble, en Européens

45 Discours de Salvador de Madariaga à la conférence européenne de la culture (Lausanne, 8 décembre 1949), op. cit.

${ }^{46}$ Congress of Europe, The Hague. Plenary Session. Sunday 9th May, 1948, morning 9am. Chairman: Mme. Pierre Brossolette. Plenary Session-Consideration of Resolution from Cultural Committee. ME-446 Actas de la Comisión Cultural en La Haya (9 de mayo), Congreso de La Haya, AHUE, p. 5. 
pour faire l'Europe, nous fassions l'Europe, presque sans nous rendre compte $^{47}$.

Las palabras pronunciadas por SdM en esta sesión del Congreso de Europa y, especialmente, la mención a la existente pero insuficiente solidaridad de hecho sin la solidaridad subjetiva, establecen una más que evidente distancia entre el el proyecto de nuestro protagonista y el que iniciará dos años después el Ministro Francés de Asuntos Exteriores, Robert Schuman, en París: Europa no se hará de golpe ni en una construcción de conjunto: se hará mediante realizaciones concretas, creando primero una solidaridad de hecho ${ }^{48}$.

La Declaración Schuman negará la existencia de la solidaridad de hecho que SdM ve ya establecida en La Haya, y pretenderá empezar a crearla con la puesta en común del carbón y el acero franco-alemán, precisamente el tipo de receta que SdM ve como inútil sin la creación previa de una consciencia y espíritu europeos si lo que se pretende es verdaderamente construir Europa sobre bases sólidas. Es más, la Declaración Schuman no hace ni tan solo referencia a la cultura, el elemento central de las ideas de SdM, ni a la necesidad de crear una solidaridad subjetiva. Y es que ambas ideas no pueden asentarse sobre bases más dispares. No debe olvidarse que lo que Robert Schuman y Jean Monnet pretenden no es construir Europa como un fin en si mismo sino resolver un problema de competitividad de la siderurgia francesa utilizando la europeización del problema como medio a la vez que se satisfacían los objetivos de los Estados Unidos de ver a los estados de Europa iniciar su integración superando las fronteras nacionales ${ }^{49}$.

Para Monnet y Schuman la solidaridad de hecho es la garantía de la paz. SdM cree que la solidaridad de hecho existe desde hace décadas, en el entramado de relaciones de todo tipo entre las naciones de Europa, lo que no ha impedido las dos últimas tragedias mundiales. Este precedente, precisamente, es el que recordará en la sesión de clausura del Congreso, el día 10 de mayo: Nous avons déjà bâti de grandes maisons internationales

47 Congress of Europe, The Hague. Plenary Session. Sunday 9th May, 1948, morning 9am. Chairman: Mme. Pierre Brossolette. Plenary Session-Consideration of Resolution from Cultural Committee. ME-446 Actas de la Comisión Cultural en La Haya (9 de mayo), Congreso de La Haya, AHUE, p. 6.

${ }_{48}$ Declaración de Robert Schuman, Ministro de Asuntos Exteriores de Francia, 9 de mayo de 1950, en Truyol y Serra, 1999, pp. 175-177.

49 Ver Gavín, 2007. 
qui se sont effondrées, non pas certes parce que les fondations n'étaient pas fermes, mais parce que ces maisons étaient vides, vides de foi ${ }^{50}$.

Y ahí está la clave para el éxito de las instituciones europeas que urge crear. La fe europeísta de las gentes que las habiten y dicha fe hay que crearla primero por qué no existe. Hay que aspirar al día que:

les Espagnoles diront «Notre Chartres», lorsque les Anglais diront «Notre Cracovie», lorsque les Italiens diront «Notre Copenhague», lorsque les Allemands diront «Notre Bruges» et reculeront d'horreur à la pensée d'y porter encore à nouveau des mains meurtrières. Cette Europe alors vivra car c'est alors que l'esprit qui dirige l'histoire aura prononcé les mots créateurs : «Fiat Europa »..$^{51}$

Una vez finalizado el Congreso de Europa no había duda acerca del valor simbólico del mismo, pero como había advertido SdM, su importancia real dependería de la asunción o no sus recomendaciones por parte de los gobiernos interesados. Centrándonos en el caso del comité cultural presidido por nuestro protagonista, la recomendación consistía en el establecimiento de un Centro Europeo para la Cultura y un Instituto Europeo para la Infancia y la Juventud entendiendo, como se ha visto en las líneas precedentes, que sin un «suplemento» cultural y espiritual cualquier proyecto de integración nacería sin vida y sin alma. El Centro Europeo para la Cultura se creó en 1950 en Ginebra, liderado por el suizo Denis de Rougemont. El nuevo centro fue establecido por el Movimiento Europeo, organización fundada el 25 de octubre de 1948 como sucesora del Comité Internacional de Movimientos para la Unidad Europea (ICMEU) establecido un año antes. Su primer Secretario General fue Joseph Rettinger e incluyó a un comité de exilados españoles. SdM fue el presidente de su comité cultural dando así continuidad a la posición que había ostentado en La Haya.

En diciembre de 1949 el comité presidido por SdM organizó una Conferencia Europea sobre la Cultura en Lausana de la cual surgió una nueva institución a la que SdM estuvo vinculado durante 22 años (1950-1972) como presidente de su consejo administrativo: el Colegio de Europa en Brujas. Establecido en 1950 abrió sus puertas con el holandés Henry

50 Congrès de l'Europe. La Haye-Mai 1948. Séance de clôture du 10 Mai 1948. M. Salvador de Madariaga (Espagne), ME-311 Correspondencia con Madariaga (19481955), Sección Cultural Internacional, AHUE.

51 Ídem. 
Brugmans como rector. La función de la nueva institución era la que había esbozado en ¡Ojo Vencedores! cinco años antes como se ha indicado anteriormente. Ofrecer a un selecto grupo de estudiantes europeos una formación genuinamente europea que complementara la compartimentada educación que hubieran recibido hasta aquel momento en sus estados respectivos en el campo de las ciencias humanas. En otras palabras, formar a la élite encargada de dotar de «alma y espíritu europeos» a las instituciones europeas creadas y por crear.

SdM había definido perfectamente el objetivo en un discurso pronunciado durante la Conferencia Europea de la Cultura en Lausana:

Si l'on évoque ensemble les deux idées France - Université, il en sort la Sorbonne ; ou Angleterre - Université, il en sort Oxford ; mais si l'on évoque ensemble les idées Europe -Université, il en sort le vide. Or l'Université est la pépinière des élites nationales et le foyer du patriotisme. Si nous voulons une Europe il faudra bien un patriotisme et un corps d'élite européens ; il faudra donc bien une Université (...) C'est vers ce but que s'oriente le projet de Collège d'Europe (...). ${ }^{52}$

O cinco años después en una alocución radiofónica:

El Colegio de Europa no es pues una universidad. Es ante todo un colegio-residencia donde cuarenta europeos de una veintena de naciones aprenden a vivir juntos, a estudiar juntos problemas de Europa y de este modo positivo a europeizarse. ${ }^{53}$

Formar a una élite europea en el espíritu europeo por encima de los compartimentos, físicos y mentales, que suponen los estados europeos para dotar de contenido y vida a las instituciones europeas podemos considerarlo la encarnación de la solución a los problemas de Europa tal y como los entiende Salvador de Madariaga. Para un hombre que por su condición de exiliado no podía dedicarse a la política en representación de la España oficial, ello representaba el máximo a lo que podía aspirar en la construcción de Europa. Si además coincidía con sus ideas, el círculo quedaba cerrado.

52 Discours de Salvador de Madariaga à la conférence européenne de la culture (Lausanne, 8 décembre 1949), op. cit.

53 Radiodiffusion Française, Salvador de Madariaga, 9 de marzo de 1955. ME-311 Correspondencia con Madariaga (1948-1955), Sección Cultural Internacional, AHUE. 
Fue como premio a esta trayectoria, a la que podríamos añadir su antifranquismo el cual tendría como punto álgido el llamado «Contubernio de Munich» en $1962^{54}$ durante una conferencia del Movimiento Europeo en dicha ciudad alemana, que recibió el Premio Carlomagno en 1973. De la ceremonia de entrega aquel día de mayo de hace casi medio siglo quedan dos discursos que, seguramente, llenaron de satisfacción a un personaje como SdM. Para alguien que se definía ante todo como un intelectual y un escritor, las palabras del político liberal alemán Walter Scheel, indicando que aquel no era un premio a la España política sino a la España literaria, no podían ser otra cosa que un reconocimiento a la personalidad de SdM por parte de un compañero de ideología ${ }^{55}$.

El elogio que le dedica Henry Brugmans es un verdadero reconocimiento a una trayectoria:

During his life this man was a technician, diplomat, and author. He can be characterized in particular by brilliance, foresightedness and vitality. Based on firm conviction he is also a European world citizen, a liberal and a federalist. He is all these things not alternately but at the same time. Therefore, I call him the provisional last Renaissance man, a «homo universale». [...] You, my dear friend Don Salvador, are the founding president of the Europe College. [...] You dreamed of Brügge becoming the European Oxford. ${ }^{56}$

Finalmente, Salvador de Madariaga en su discurso de aceptación hizo gala de su personal europeísmo en base a la bandera de la Comunidad Económica Europea (CEE) con doce estrellas doradas en círculo sobre fondo azul. Para él tal bandera tal y como está no significa nada. Propone mantener las estrellas y el color azul, pero distribuyéndolas de otra manera:

I suggest that we keep the blue background and the golden stars; but the stars should be depicted where every European capital city is lo-

${ }^{54}$ Sobre el Contubernio de Munich ver: Amat, 2016; de Pedro y Solé, 1999; Satrustegui et al., 1993.

55 Entrega del Premio Carlomagno a Salvador de Madariaga, $A B C, 1$ de junio de 1973, p. 37 [consultada en la Hemeroteca on-line de $A B C$ : https://www.abc.es/archivo/periodicos/abc-madrid-19730601-61.html, el 27 de octubre de 2020].

${ }^{56}$ Laudation (extract) speaker Hendrik Brugmans on the laureate. Der Internatioale Karlspreis zu Aachen. Für die Einheit Europas, en http://www.karlspreis.de/en/laureates/ don-salvador-de-madariaga-1973/laudation-extract-by-hendrik-brugmans [página web visitada el 27 de octubre de 2020]. 
cated without showing the contours of the map. Thus, the stars become symbolic representation of the nations whose borders have disappeared. This flag would have a meaning. It would be a constellation with its own special character. New stars would be added when new members joined the alliance. And so, with every step forward towards our goal we would see a new star light up on the blue firmament of Europe's flag. ${ }^{57}$

Tal propuesta no era nueva. SdM la había propuesto ya en 1951 desde Oxford $^{58}$. Y es que para el personaje objeto de este texto, diseño eficiente sin significado no tenía sentido, Europa sin la diversidad de sus naciones no era nada y las fronteras no eran otra cosa que barreras que mantenían a los europeos compartimentados e impedían el surgimiento de una auténtica solidaridad europea.

\section{Fuentes}

Archivos Históricos de LA Unión EuRoPea (AHUE), Firenze, Italia.

\section{Bibliografía}

Amat, Jordi, La primavera de Múnich: Esperanza y fracaso de una transición democrática, Barcelona, Tusquets, 2016.

BECKER, Carl L., How new will the better world be? A discussion of post-war reconstruction. New York, Alfred A. Knopf, 1944.

Beneyto, José María, Tragedia y razón. Europa en el pensamiento español del siglo XX, Madrid, Taurus, 1999.

Beneyto, José María, «Estudio Introductorio» en De Madariaga, Salvador, Bosquejo de Europa, Madrid, Ediciones Encuentro, 2010, pp. 5-33.

57 Speech (extract) by Don Salvador de Madariaga, Der Internatioale Karlspreis zu Aachen. Für die Einheit Europas, en http://www.karlspreis.de/en/laureates/don-salvadorde-madariaga-1973/speech-extract-by-don-salvador-de-madariaga [página web visitada el 27 de octubre de 2020].

58 Proposal for a European flag by Salvador de Madariaga (1 December 1951), en The European Navigator: https://www.cvce.eu/en/obj/proposal_for_a_european_flag_by_salvador_de_madariaga_1_december_1951-en-fc21dbed-deaf-4c0d-970a-d1d920cd7467. html [página web visitada el 27 de octubre de 2020]. 
Coudenhove-Kalergi, Richard N., Crusade for Pan-Europe. Autobiography of a man and a movement, New York, Putnam's Sons, 1943.

Coudenhove-Kalergi, Richard N., Pan-Europa, Madrid, Ediciones Encuentro, 2010.

De Madariaga, Isabel, «S. de Madariaga et le Foreign Office. Un episode d'histoire diplomatique. Juillet-décembre 1936», Revista de Estudios Internacionales, vol. 4, n. ${ }^{\circ}$ 2, abril-junio de 1983, pp. 229-257.

De Madariaga, Salvador, Discursos Internacionales, Madrid, M. Aguilar, 1934.

De Madariaga, Salvador, Anarquía o jerarquía. Ideario para la constitución de la tercera república, Madrid, M. Aguilar, 1935.

De Madariaga, Salvador, ;Ojo, Vencedores!, Buenos Aires, Editorial Sudamericana, 1945.

De Madariaga, Salvador, Bosquejo de Europa, México DF, Editorial Hermes, 1951.

De Madariaga, Salvador, General, Márchese usted, Nueva York, Ediciones Ibérica, 1959.

De Madariaga, Salvador, Bosquejo de Europa, Madrid, Ediciones Encuentro, 2010.

DE PEDRo, Pilar y SolÉ, Queralt, 30 anys d'història d'europeisme català 19481978. El contuberni de Munic, Barcelona, Mediterrània, 1999.

FARnetTi, Paolo Bertella, Gli Stati Uniti e l'Unità Europea 1940-1950. Percorsi di un'idea. Milano, Franco Angeli, 2004.

Galambos, Louis (ed.), The papers of Dwight David Eisenhower. Vol. XII: NATO and the campaign of 1952, Baltimore, The John Hopkins University Press, 1989.

Gavín, Víctor, Europa Unida. Orígenes de un malentendido consciente, Barcelona, Publicacions i edicions de la Universitat de Barcelona, 2007.

GonzÁlez Cuevas, Pedro Carlos, «Salvador de Madariaga, pensador político», Revista de Estudios Políticos (nueva época), n. ${ }^{\circ}$ 66, octubre-diciembre de 1989, pp. 145-181.

KAPLAN, Lawrence S, The United States and NATO. The formative years, Lexington, The University Press of Kentucky, 1984.

Lago, José Ramón y Domínguez CASTRo, Luis, «Entre Oxford y Lausana. Salvador de Madariaga y los orígenes de la educación para la ciudadanía en Europa (1936-1949)» [comunicación presentada en el seminario Euclio Escuela y Europa: Hacer las Europas. Identidades, europeización, proyección exterior y relato nacional español en el proceso de integración europea, celebrado en la Facultad de Geografía e Historia de la Universidad Complutense de Madrid el 6 de Octubre de 2017, en el marco de los equipos de investigación HAR2015-64429-C1-1-P (MINECO/FEDER) y HAR201564429-C2-2-P (MINECO/FEDER)].

LIPGENS, Walter, Documents on the History of European Integration (4 vols.), Berlin y Nueva York, Walter de Gruyter, 1986. 
Ortega y Gasset, José, La rebelión de las masas y otros ensayos, Madrid, Alianza Editorial, 2014.

Ortega y Gasset, José, Meditación de Europa y otros ensayos, Madrid, Alianza Editorial, 2015.

PAzos, Antón M., « My Dear de Madariaga: correspondencia entre Madariaga e Eden en 1936 en pro dunha paz negociada na Guerra Civil Española / My Dear de Madariaga: correspondence Madariaga - Eden in 1936 to promote a negotiated peace in the Spanish Civil War», Cuadernos de Estudios Gallegos, LVI, n. ${ }^{\circ} 122$, enero-diciembre de 2009, pp. 317-332.

Pérez Garzón, Juan Sisinio, «Modernización y europeización en el pensamiento español de la segunda mitad del siglo xx: hacia el fin de las angustias regeneracionistes», en Gómez Benito, Cristóbal (coord.), Joaquín Costa y la modernización de España, Madrid, Congreso de los Diputados, 2011, pp. 199-237.

Preston, Paul, «Salvador de Madariaga. Un quijote en la política», en PresTON, Paul, Las tres Españas del 36, Barcelona, Random Housse Mondadori, 1999, pp. 193-226.

Preston, Paul, Las tres Españas del 36, Barcelona, Random House Mondadori, Barcelona, 1999.

Satrustegui, Joaquín et al., Cuando la transición se hizo posible. El Contubernio de Múnich, Madrid, Tecnos, 1993.

Sobrino, José Manuel, «La actualidad de las aportaciones de Salvador de Madariaga a la idea de Europa», Anuario da Facultade de Dereito da Universidade da Coruña, n. ${ }^{\circ}$ 7, 2003, pp. 759-775.

STREIT, Clarence K, Union Now. A proposal for a federal union of the leading democracies, New York, Harper and Brothers publishers, 1940.

TAmayo Barrena, Ana María, «España ante el Pacto Briand-Kellogg», Cuadernos de Historia Moderna y Contemporánea, vol. 5, 1984, pp. 187-213.

The Federal Union, Uncommon Sense. A pamphlet for now. 1940. Consultable en la página web de The Federal Union: http://www.federalunion.org.uk/ wp-content/uploads/2010/08/uncommonsense.pdf [página web consultada el 27 de octubre de 2020].

Thomas, Elbert D., The Four Fears. Chicago: Ziff-Davis Publishing Company, 1944.

TRUYOl y SERRA, Antonio, La Integración Europea. Análisis histórico-institucional con textos y documentos. (I) Génesis y desarrollo de la Comunidad Europea (1951-1979), Tecnos, Madrid, 1999.

VAN DOREN, Carl, The great rehearsal. The story of the making and ratifying of the Constitution of the United States, New York, The Viking Press, 1948.

Woods, Randall Bennett, Fulbright. A biography, Cambridge, Cambridge University Press, 1995. 
Wooley, Wesley T., Alternatives to Anarchy. American supranationalism since World War II, Bloomington, Indiana University Press, 1988.

WynNer, Edith y LLOYD, Georgia (eds.), Searchlight on peace plans. Choose your road to World Government, New York, E.P. Dutton and Company, Inc. 1949.

\section{Financiación}

Este texto forma parte del proyecto de investigación HAR201564429-C2-1-P (Mineco /Feder): Hacer las Europas: identidades, europeización, proyección exterior y relato nacional español en el proceso de integración europea.

\section{Datos del autor}

Víctor Gavin Munté, profesor agregado permanente de la Sección de Historia Contemporánea y Mundo Actual, Departamento de Historia y Arqueología, Facultad de Geografía e Historia de la Universidad de Barcelona. Ha publicado extensamente sobre la historia de la integración europea y de las relaciones Europa-Estados Unidos. Es miembro de los proyectos de investigación: Europeísmo y redes transatlánticas en los siglos XX y XXI (PGC2018-095884-B-C21 y PGC2018095884-B-C22); España y Portugal ante la segunda ampliación de las Comunidades Europeas. Un estudio comparado 1974-1986 (HAR2017-84957-P) y del Grupo de Investigación consolidado del Centro de Estudios Históricos Internacionales (CEHI) de la Universidad de Barcelona (GREC-CEHI SGR 2017-1425). Es también miembro del Editorial Board del Journal of Transatlantic Studies y de los consejos de redacción de las revistas Pasado y memoria. Revista de Historia Contemporánea e Índice Histórico Español. 